American Journal of Pharmaceutical Education 2021; 85 (4) Article 7928.

\title{
BRIEF
}

\section{Assessment of Cultural Competence in Pharmacy Students Prior to Advanced Pharmacy Practice Experiences}

\author{
Shadi Doroudgar, PharmD, ${ }^{\mathrm{a}, \mathrm{b}}$ Baovy Dang, PharmD, ${ }^{\mathrm{a}}$ Haiyen Nguyen, PharmD, ${ }^{\mathrm{a}}$ Rae R. Matsumoto, PhD ${ }^{\mathrm{a}}$ \\ ${ }^{a}$ Touro University California, College of Pharmacy, Vallejo, California \\ ${ }^{\mathrm{b}}$ Stanford University, School of Medicine, Stanford, California \\ Submitted November 14, 2019; accepted December 15, 2020; published April 2021.
}

\begin{abstract}
Objective. To assess various aspects of cultural competence in second year Doctor of Pharmacy students' and investigate the relationship between cultural competence and students' demographics, work experience, and prior education.

Methods. A 63-item survey modified from the Clinical Cultural Competency Questionnaire (CCCQ) and comprising four domains (knowledge, skills, encounters or situations, and attitudes towards cultural competency) was administered to second year pharmacy students before they started their advanced pharmacy practice experiences (APPEs). Additional questions regarding their ability to identify and recognize elements of cultural competence were asked. The effects of demographics, work experience, and education on cultural competence also were assessed.

Results. Ninety-seven students $(86.6 \%)$ participated in the study. The majority of participants were Asian, female, and in their late 20 s. Most students agreed or strongly agreed that they could identify and recognize elements of cultural competence. However, participants indicated they were only a little or somewhat comfortable when asked questions about knowledge, skills, and comfort. Students indicated they had "quite a bit" of competence regarding attitudes towards other cultures. Previous cultural diversity training in undergraduate studies and pharmacy school were associated with higher scores on the modified CCCQ.

Conclusion. The findings emphasize the importance of schools providing training in the didactic and experiential portion of the pharmacy curriculum to increase pharmacy students' knowledge, skills, comfort, and attitudes towards other cultures.
\end{abstract}

Keywords: cultural competence, pharmacy, pharmacy students

\section{INTRODUCTION}

The US population, known as a "melting pot," is extremely diverse. ${ }^{1}$ The population increases by one international migrant every 46 seconds. $^{2}$ This trend is expected to continue over time. ${ }^{3}$ The diversity of culture, race, and language creates new opportunities and challenges for healthcare providers in delivering equitable services. ${ }^{4,5}$ Differences in beliefs, cultures, and/or languages between patients and healthcare providers could lead to misunderstandings that negatively impact patients, including limiting their access to care, threatening their safety, and causing their dissatisfaction. ${ }^{6,7}$ To effectively care for the growing diverse patient population, healthcare providers must practice cultural competence, which is defined as "effective delivery of culturally and linguistically appropriate service in cross-cultural settings.", 7,8

Corresponding Author: Shadi Doroudgar, Touro University California, College of Pharmacy, 1310 Club Dr., Vallejo, CA 94592. Tel: 818-281-5856. Email: shadi.doroudgar@tu.edu
Cultural competence in healthcare professionals, such as nurses and physicians, has been studied. ${ }^{8-13}$ In these fields, the importance of integrating cultural competence into the educational curriculum is reflected in improved provider outcomes, healthcare access, utilization outcomes, and patient outcomes. ${ }^{7,9-13}$ In contrast, cultural competence has relatively recently been recognized in pharmacy practice and education as it was first incorporated into the Accreditation Council for Pharmacy Education (ACPE) Standards 2006. ${ }^{14}$

Studies of the education and practice of cultural competence in pharmacy students and pharmacy healthcare providers suggest benefits. However, many questions remain regarding validation of improved outcomes and optimization of curricula and training elements. Among cultural competence assessment tools studied, ${ }^{15-19}$ the Clinical Cultural Competency Questionnaire (CCCQ), developed by the Center for Healthy Families and Cultural Diversity in the Department of Family Medicine and 


\section{American Journal of Pharmaceutical Education 2021; 85 (4) Article 7928.}

Community Health at Rutgers Robert Wood Johnson Medical School, has been validated in several studies that assessed cultural competence in pharmacy students. ${ }^{20-22}$ Because of its relevance to pharmacy student education and previous validation in studies involving pharmacy students, the CCCQ was selected for use in this study. The CCCQ measures different aspects of cultural competency, including knowledge of cultural competency, skills in dealing with sociocultural issues, comfort levels in handling cross-cultural encounters/situations, attitudes towards health disparities and sociocultural issues, self-awareness of one's own culture, and importance of cultural diversity training in health professionals. This study was different from prior studies as it assesses cultural competence knowledge and self-awareness of Doctor of Pharmacy (PharmD) students prior to beginning their advanced pharmacy practice experiences (APPEs).

The purpose of this study, which was conducted at the Touro University California College of Pharmacy (TUC-COP), was to assess the knowledge of cultural competence, skills in dealing with sociocultural issues, comfort in handling cross-cultural encounters, and attitudes towards health disparities in second professional year (P2) pharmacy students prior to beginning their APPEs, and to investigate the relationship between cultural competence and students' demographics, work experience, education, and prior training. The college has a " $2+2$ " PharmD program, meaning that students completed all didactic coursework during the first and second years of the curriculum, followed by two years of APPEs.

\section{METHODS}

A cross-sectional study design was used to assess the cultural competence of P2 students (class of 2020) at TUC-COP from March 2018 to May 2018. The only inclusion criterion was any $\mathrm{P} 2$ student willing to participate in the survey. Exclusion criteria were being unwilling to participate or refusing to consent to participating in the study.

The CCCQ is a 63-item survey developed to assess physician's knowledge, skills, and attitudes related to cultural competency and health disparities. ${ }^{23}$ For this study, we obtained permission from the authors to modify the CCCQ to be more relevant to pharmacy students. The version we modified for pharmacy students will be referred to as the CCCQ-Modified Pharmacy (CCCQ-MP).

The CCCQ-MP had four areas of focus or domains. The first domain assessed students' knowledge of cultural competency, including health disparities experienced by diverse groups, sociocultural issues, different healing traditions, and the Office of Minority Health's national standards for Culturally and Linguistically Appropriate Services (CLAS) in Health Care. The second domain assessed students' skills in dealing with sociocultural issues and focused on culturally sensitive patient care, patient education, counseling, and dealing with crosscultural conflicts relating to treatment and adherence. The third domain assessed students' comfort with handling cross-cultural encounters/situations and their comfort level in dealing with cross-cultural situations that may be faced as a pharmacist working with different ethnic groups. Examples included caring for patients with limited English proficiency and those from culturally diverse backgrounds. The fourth domain assessed students' attitudes towards health disparities including contributing factors, interactions with patients, and self-awareness of cultural differences in patient care. The following point values were assigned to the six answer choices: do not know $=0$, not at all $=1$, a little $=2$, somewhat $=3$, quite a $b i t=4$, and very $=5$. The cultural competency score for each participant was determined by adding together the points for all of a students' answer choices. Mean scores for each domain were calculated by adding the responses to the items and dividing the sum by the number of items in each component, as done in prior studies that used the CCCQ. ${ }^{21,24}$ The total mean score was calculated for each participant by adding the scores of the responses to all items and dividing the sum by 63 , the total number of all items in the survey.

Additionally, the participants were asked two questions from the American Association of Colleges of Pharmacy (AACP) Graduating Student Survey. These questions asked about "the degree to which you agree or disagree with whether your Pharm.D. curriculum prepared you to (1) identify cultural disparities in healthcare, and (2) recognize and address cultural disparities in access to and delivery of healthcare." The participants were asked to select one of the following responses: unable to comment, strongly disagree, disagree, agree, and strongly agree. These items were not assigned a score and not counted as part of the CCCQ-MP.

The survey was administered online using Qualtrics XM (Qualtrics). An email invitation was sent to all P2 students in March 2018. Reminders were sent out in April 2018. The students participated in the study from March 2018 to May 2018.

All statistical analyses were conducted using STATA/IC, version 14.2 (StatCorp, LLC). Continuous data are reported as mean and standard deviation and categorical data are reported as numbers and percentages of respondents. A multivariate linear regression 


\section{American Journal of Pharmaceutical Education 2021; 85 (4) Article 7928.}

was conducted to study the effects of variables on the cultural competence score.

\section{RESULTS}

Of the 97 P2 students from the class of 2020, 84 completed the survey ( $86.6 \%$ response rate). The participants had a mean age of 27 years (SD 3.6) and most were female (64\%). Participant demographics are summarized in Table 1. The majority of the students were Asian (71.4\%). The remainder were Latinx/Hispanic (10.7\%), Caucasian (6\%), and African American (2.4\%). Half of the students indicated they had received either a little (29.8\%) cultural competence training or none (20.2\%) during undergraduate education.
The participants' scores from the CCCQ-MP are summarized in Table 2 . The overall mean score for cultural competence was $3.2(0.3)$, indicating a familiarity between "somewhat" to "quite a bit." The knowledge of cultural competence, skills in dealing with sociocultural issues, and comfort in handling cross-cultural encounters yielded mean scores of $2.9(0.3), 2.6(0.5)$, and $2.7(0.4)$, respectively, indicating that most students believed they were "a little" to "somewhat" competent. The attitudes towards health disparities domain, which included self-awareness, had the highest overall mean of all domains with a score of $4.1(0.2)$, indicating "quite a bit" of competence.

The participants expressed confidence when answering the two questions from the AACP Graduating

Table 1. Demographics of Second Year Pharmacy Students Who Participated in an Assessment of Cultural Competence Prior to Starting Advanced Pharmacy Practice Experiences

\begin{tabular}{|c|c|c|}
\hline Participants $(\mathrm{N}=84)$ & M (SD) & Frequency $(\%)$ \\
\hline Response rate & & $84(86.6)$ \\
\hline Age & $27(3.6)$ & \\
\hline \multicolumn{3}{|l|}{ Gender } \\
\hline Female & & $54(64)$ \\
\hline Male & & $30(36)$ \\
\hline \multicolumn{3}{|l|}{ Ethnicity } \\
\hline Asian American & & $60(71.4)$ \\
\hline Latinx/Hispanic & & $9(10.7)$ \\
\hline Caucasian & & $5(6.0)$ \\
\hline African American/Black & & $2(2.4)$ \\
\hline Native Hawaiian/Other Pacific Islander & & $1(1.2)$ \\
\hline Two or more ethnicities & & $6(7.1)$ \\
\hline Other & & $1(1.2)$ \\
\hline \multicolumn{3}{|l|}{ Visited or lived in foreign country(s) } \\
\hline Yes & & $60(71.0)$ \\
\hline No & & $24(29.0)$ \\
\hline \multicolumn{3}{|c|}{ Total cultural competence training in undergraduate } \\
\hline None & $0.6(1.2)$ & $17(20.2)$ \\
\hline A Little & $0.9(1.4)$ & $25(29.8)$ \\
\hline Some & $0.8(1.4)$ & $21(25.0)$ \\
\hline Quite A Bit & $0.6(1.3)$ & $14(16.7)$ \\
\hline A Lot & $0.3(1.0)$ & $7(8.3)$ \\
\hline \multicolumn{3}{|c|}{ Total years of work experience prior to pharmacy } \\
\hline 0 Years & $0.4(1.0)$ & $11(13.1)$ \\
\hline 1-5 Years & $1.7(1.6)$ & $46(54.8)$ \\
\hline $6-10$ Years & $0.8(1.4)$ & $21(25)$ \\
\hline 11-15 Years & $0.1(0.7)$ & $3(3.6)$ \\
\hline$>15$ Years & $0.1(0.5)$ & $3(3.6)$ \\
\hline \multicolumn{3}{|c|}{ Years of healthcare work experience prior to pharmacy } \\
\hline 0 Years & $1.0(1.4)$ & $27(32.1)$ \\
\hline 1-5 Years & $2.1(1.6)$ & $54(64.3)$ \\
\hline $6-10$ Years & $0.1(0.4)$ & $2(2.4)$ \\
\hline 11-15 Years & $0.0(0.0)$ & $0(0.0)$ \\
\hline$>15$ Years & $0.0(0.3)$ & $1(1.2)$ \\
\hline
\end{tabular}




\section{American Journal of Pharmaceutical Education 2021; 85 (4) Article 7928.}

Table 2. Assessment Scores of Second Year Pharmacy Students Who Participated in an Assessment of Cultural Competence

\begin{tabular}{ll}
\hline Areas of Cultural Competence $^{\text {a }}$ & M (SD) \\
\hline Overall & $3.2(0.3)$ \\
Knowledge of cultural competence & $2.9(0.3)$ \\
Skills in dealing with sociocultural issues & $2.6(0.5)$ \\
$\quad \begin{array}{l}\text { Comfort in handling cross-cultural } \\
\quad \text { encounters }\end{array}$ & $2.7(0.4)$ \\
$\quad$ Attitudes towards health disparities & $4.1(0.2)$ \\
\hline${ }^{a}$ Assessment scores: $0=$ do not know, 1=not at all, 2=a little, \\
$3=$ somewhat, 4= quite a bit, 5=very
\end{tabular}

Student Survey. Two-thirds (66.7\%) of the students selected they agreed or strongly agreed that they were able to identify cultural disparities in healthcare. Additionally, $65.5 \%$ also agreed or strongly agreed that they would be able to recognize and address cultural disparities in access to and delivery of healthcare.

The results of the multivariate linear regression analysis are shown in Table 3. The effects of age, gender, race, whether the participant had lived in a foreign country, total years of work experience prior to pharmacy school, total years of work experience in healthcare prior to pharmacy school, and cultural diversity education in undergraduate studies, pharmacy school, and other graduate studies on cultural competence were investigated. Only cultural diversity training in undergraduate education (95\% CI [0.022-0.18]; $p=.013)$ and in pharmacy school (95\% CI $[0.13-0.35] ; p<.001)$ were associated with higher CCCQ-MP scores.

\section{DISCUSSION}

In this study, the cultural competence of P2 pharmacy students prior to starting their APPEs was assessed using the CCCQ-MP. Additionally, correlations of demographics with cultural competence were investigated.
The majority of the participants were in their late 20s, which is somewhat older than most students who participated in other published studies on cultural competence. $^{22,24}$ Older students may be more comfortable when dealing with cross-cultural situations and issues, perhaps because of increased maturity. The majority of participants identified as Asian American. This difference in demographics compared to that of previous studies that involved mostly Caucasian and/or African American students may have had some impact on the students' cultural competency. ${ }^{20-22}$

Participants' answers to the two questions on the AACP Graduating Student Survey differed from the answers provided on the more comprehensive and detailed CCCQ-MP. Approximately two-thirds of the participants believed they could identify cultural disparities in healthcare, as well as recognize and address cultural disparities in access to and delivery of healthcare. However, the CCCQ-MP scores averaged across all four domains indicated the participants had only a minimal or moderate level of cultural competence as most of their responses were in the 'somewhat' category. . None of the respondents scored the maximum points in the four domains, suggesting an opportunity for improvement in cultural competence training. The need for formal education regarding this topic cannot be overlooked, even in students who are culturally diverse.

The CCCQ-MP allowed us to assess students' competence in individual domains. The mean scores for the three domains of knowledge, skills, and encounters $(2.9,2.6,2.7$, respectively) fell between the responses for being "a little" and "somewhat" culturally competent, suggesting that the majority of participants needed more training in these domains. A low score in these domains prior to starting APPEs was expected as the students had not yet had extensive exposure to clinical practice. Students' attitudes toward health disparities had the highest

Table 3. Effects of Demographics on Cultural Competence in Second Year Pharmacy Students Who Participated in an Assessment of Cultural Competence

\begin{tabular}{lccc}
\hline Areas of Cultural Competence & Coefficient & 95\% Confidence Interval & $\boldsymbol{p}$ value \\
\hline Age & -0.007 & {$[-0.037-0.022]$} & .62 \\
Gender & 0.068 & {$[-0.12-0.25]$} & .47 \\
Race & 0.013 & {$[-0.066-0.092]$} & .75 \\
Lived in a foreign country & 0.077 & {$[-0.12-0.27]$} & .44 \\
Undergraduate education training on cultural competence & 0.10 & {$[0.022-0.18]$} & $.013^{\mathrm{a}}$ \\
Pharmacy school training on cultural competence & 0.24 & {$[0.12-0.35]$} & $<.001^{\mathrm{a}}$ \\
Graduate education on cultural competence & 0.074 & {$[-0.008-0.16]$} & .078 \\
Total work experience & 0.023 & {$[-0.006-0.052]$} & .13 \\
Work experience in a health care field & -0.008 & {$[-0.054-0.039]$} & .74 \\
\hline
\end{tabular}

${ }^{a} p$ value is defined as $<.05$, between the undergraduate training and the cultural competence assessment scores in each domain 


\section{American Journal of Pharmaceutical Education 2021; 85 (4) Article 7928.}

assessment scores among the four domains. The majority of respondents ranked themselves as being "quite a bit" to "very" culturally competent toward health disparities. The higher ratings in this domain may have been because some of the questions in this section related to students' self-awareness and identifying contributing factors to disparities.

The results of the multivariate regression analysis highlighted the importance of cultural competence training in achieving a higher score on the CCCQ-MP. Greater undergraduate training and pharmacy school training in cultural diversity were associated with higher cultural competence assessment scores. Interestingly, the other parameters studied did not have a significant impact on cultural competence scores per CCCQ-MP. Perhaps because of the vast cultural differences among pharmacy students in the United States and around the world, the only variable that can accurately predict competence is students having formal training in this area. At the time of this study, cultural competence was not extensively covered as a standalone topic in the TUC-COP didactic curriculum. Instead, elements of cultural competence were incorporated throughout the didactic curriculum in various courses. Since then, a more formal hour-long lecture session and approximately three hours of reading assignments on cultural competence have been incorporated into the TUC-COP curriculum during the first year to provide students with an earlier, formal introduction to the topic. The findings thus stress the importance of structured education in cultural competence, even in environments where there is extensive interactions with diverse populations.

The findings of this study are consistent with those from prior studies: there is a need to integrate cultural competence into the PharmD curriculum. ${ }^{25-27}$ Prior studies, including one by Haack in 2008, have shown that adding curriculum content on cultural competence leads to greater cultural competency among pharmacy students. ${ }^{28}$ In the last 10 years, several studies have assessed achievement of the ultimate goal of cultural competence training in students, ie, cultural competence in the pharmacy profession. ${ }^{28-31}$ However, work is still needed to define and assess effective curricular approaches to teaching cultural competence, which should incorporate both didactic and experiential components, along with self-assessments.

This study has some limitations. The study participants consisted of mostly Asian female students completing their second year of pharmacy school prior to beginning their APPEs. As such, the findings may not be generalizable to all pharmacy students across the United States. Perhaps surveying other regions of the United States would provide further insights into training needs.
Additionally, the second AACP Graduating Student Survey question attempted to assess four different areas in the same question, posing limitations in interpreting responses to this question. In future assessments, this question could be separated into four distinct sections to more accurately capture student answers.

In future research, reassessing the same pharmacy students after they complete their APPEs may be worthwhile as the " $2+2$ " curriculum allows for more hands on experience during the experiential years and may contribute to greater cultural competence scores. Moreover, variables that did not predict cultural competence scores in the CCCQ-MP prior to APPEs may be more predictive following rigorous rotation experiences in which $100 \%$ of the students encounter cultural competence events. Future studies should also include direct measures of students' cultural competence in addition to measures of students' perceptions of their cultural competence.

\section{CONCLUSION}

Although the majority of pre-APPE students who participated in this study expressed confidence in identifying and recognizing elements of cultural competence, most were only "somewhat" comfortable when assessed using the multi-item CCCQ-MP. Undergraduate and pharmacy school training in cultural competence was associated with greater scores on the questionnaire. This emphasizes the importance of providing students with graduate level training in the didactic and experiential portion of the pharmacy school curriculum to increase their proficiency with regards to knowledge of, skills in, comfort with, and attitudes towards other cultures, even in diverse student populations.

\section{REFERENCES}

1. Perez AD, Hirschman $C$. The changing racial and ethnic composition of the US population: emerging American identities. Popul Dev Rev. 2009;35(1):1-51.

2. United States Census Bureau. U.S. and World Population Clock. https://www.census.gov/popclock/. Accessed March 26, 2021.

3. Census Bureau projects U.S. and world population on New Year's day. News release. United States Census Bureau; December 30, 2019. https://www.census.gov/newsroom/pressreleases/2019/

newyears2020.html\#: : text $=$ The $\% 20$ combination $\% 20$ of $\% 20$ births $\% 2 \mathrm{C} \% 20$ deaths,from $\% 20 \mathrm{New} \% 20$ Year $\% 27 \mathrm{~s} \% 20 \mathrm{Day} \% 202019$. Accessed March 26, 2021.

4. Singleton K, Krause EM. Understanding cultural and linguistic barriers to health literacy. Ky Nurse. 2010;58(4):4, 6-9.

5. Suphanchaimat R, Kantamaturapoj K, Putthasri W, Prakongsai P. Challenges in the provision of healthcare services for migrants: a systematic review through providers' lens. BMC Health Serv Res. 2015;15:390.

6. Quist RM, Law AV. Cultural competency: agenda for cultural competency using literature and evidence. Res Social Adm Pharm. 2006;2(3):420-438. 


\section{American Journal of Pharmaceutical Education 2021; 85 (4) Article 7928.}

7. O'Connell MB, Korner EJ, Rickles NM, Sias JJ. Cultural competence in health care and its implications for pharmacy. Part 1. Overview of key concepts in multicultural health care.

Pharmacotherapy. 2007;27(7):1062-1079.

8. Truong M, Paradies Y, Priest N. Interventions to improve cultural competency in healthcare: a systematic review of reviews. BMC Health Serv Res. 2014;14:99.

9. Anderson LM, Scrimshaw SC, Fullilove MT, Fielding JE, Normand J. Task Force on Community Preventive Service. Culturally competent healthcare systems. a systematic review. Am J Prev Med. 2003;24(3 Suppl):68-79.

10. Beach MC, Price EG, Gary TL, et al. Cultural competence: a systematic review of health care provider educational interventions. Med Care. 2005;43(4):356-373.

11. Hansen ND. Teaching cultural sensitivity in psychological assessment: a modular approach used in a distance education program. J Pers Assess. 2002;79(2):200-206.

12. Sorensen J, Norredam M, Dogra N, Essink-Bot ML, Suurmond J, Krasnik A. Enhancing cultural competence in medical education. Int J Med Educ. 2017;8:28-30.

13. Soule I. Cultural competence in health care: an emerging theory. ANS Adv Nurs Sci. 2014;37(1):48-60.

14. Accreditation Council for Pharmacy Education. Accreditation

Standards and Guidelines for the Professional Program in Pharmacy

Leading to the Doctor of Pharmacy Degree ("Standards 2007 v2.0").

Published February 2011. https://www.acpe-accredit.org/pdf/

FinalS2007Guidelines2.0.pdf. Accessed March 26, 2021.

15. Cultural Competence Checklist: Personal Reflection. 2010; https://www.tota.org/assets/MCC/SessionHANDOUTS/HANDOUT \%20107.pdf. Accessed March 26, 2021.

16. Cultural Competency Organizational Self Assessment (OSA) Question Bank. 2006; https://www.aidsetc.org/. Accessed March 26, 2021.

17. A Physician's Practical Guide to Culturally Competent Care. 2013; https://cccm.thinkculturalhealth.hhs.gov/videos/index.asp.

Accessed March 26, 2021.

18. Cultural \& Linguistic Competence Health Practitioner Assessment. 2010; https://www.clchpa.org/. Accessed March 26, 2021.

19. Mason JL. Cultural Competence Self-Assessment

Questionnaires: A Manual for Users. 1995; https://files.eric.ed.gov/

fulltext/ED399684.pdf. Accessed March 26, 2021.
20. Echeverri M, Brookover C, Kennedy K. Nine constructs of cultural competence for curriculum development. Am J Pharm Educ. 2010;74(10):181.

21. Echeverri M, Brookover C, Kennedy K. Assessing pharmacy students' self-perception of cultural competence. J Health Care Poor Underserved. 2013;24(1 Suppl):64-92.

22. Okoro ON, Odedina FT, Reams RR, Smith WT. Clinical cultural competency and knowledge of health disparities among pharmacy students. Am J Pharm Educ. 2012;76(3):40.

23. Clinical Cultural Competency Questionnaire (CCCQ). Center for Healthy Families and Cultural Diversity, Department of Family

Medicine, UMDNJ-Robert Wood Johnson Medical School. 2001. https://rwjms.rutgers.edu/documents/departments/Family $\%$ 20Medicine/Grants\%20-\%20projects/Pretraining.pdf. Accessed March 26, 2021.

24. VanZant S. Assessing Multicultural Knowledge, Attitudes, Skills and Comfort: Medical Education for a Changing World. 2014. https://corescholar.libraries.wright.edu/cgi/viewcontent.cgi? article $=1123 \&$ context $=\mathrm{mph}$. Accessed March 26, 2021.

25. Onyoni EM, Ives TJ. Assessing implementation of cultural competency content in the curricula of colleges of pharmacy in the United States and Canada. Am J Pharm Educ. 2007;71(2):24.

26. Cooper LA, Vellurattil RP, Quinones-Boex A. Pharmacy students' perceptions of cultural competence encounters during practice experiences. Am J Pharm Educ. 2014;78(2):31.

27. Assemi M, Cullander C, Hudmon KS. Implementation and evaluation of cultural competency training for pharmacy students. Ann Pharmacother. 2004;38(5):781-786.

28. Haack S. Engaging pharmacy students with diverse patient populations to improve cultural competence. Am J Pharm Educ. 2008;72(5):124.

29. O'Connell MBP, Korner EJ, Rickles NM, Sias JJ. Cultural competency in health care and its implications for pharmacy. part 2: emphasis on pharmacy systems and practice. Pharmacotherapy. 2009;29(2):14e-34e.

30. Poirier TI, Butler LM, Devraj R, Gupchup GV, Santanello C, Lynch JC. A cultural competency course for pharmacy students. Am J Pharm Educ. 2009;73(5):81.

31. Westberge SM, Bumgardner MA, Lind PR. Enhancing cultural competency in a college of pharmacy curriculum. Am J Pharm Educ. 2005;69(5):82. 